\title{
Size and Shape Homogenization of Ag Nanoparticles by Laser Irradiation
}

\author{
Jonghui Lim, Sungwoon Lee, and Sangwoon Yoon* \\ Department of Chemistry, Institute of Nanosensor and Biotechnology, Dankook University, Gyeonggi 448-701, Korea \\ *E-mail: sangwoon@dankook.ac.kr \\ Received July 26, 2011, Accepted September 20, 2011
}

Key Words : Ag nanoparticle, Surface plasmon resonance, Monodisperse metal nanoparticles, Laser excitation, Nanoparticle synthesis

Noble metal nanoparticles have been a subject of great interest in nanochemistry due to their unique optical properties and a wide range of applications. ${ }^{1}$ Collective oscillation of conduction electrons, referred to as surface plasmon, renders noble metal nanoparticles distinguished from other nanoscale materials. Resonance of the surface plasmon with the frequency of incident light gives rise to strong absorption and scattering of light by the nanoparticles $\left(\varepsilon \sim 10^{10} \mathrm{M}^{-1} \mathrm{~cm}^{-1}\right){ }^{2,3}$ The electromagnetic fields near the nanoparticles induced by the surface plasmon resonance (SPR) increase Raman scattering cross-sections of molecules in close proximity by 6 to 14 orders of magnitude, which is called the surface-enhanced Raman scattering (SERS). ${ }^{4}$ SERS enables detection of single molecules and has been used as a major detection scheme of biosensors., Relaxation of the excited SPR via localized heating of the surroundings provides a basis for photothermal therapy. ${ }^{7}$

The SPR of noble metal nanoparticles that makes all these possible varies with the size and shape of the nanoparticles. ${ }^{2}$ Therefore, nanoparticles with a broad distribution of size and shape have poorly defined SPR characteristics. In this regard, preparing noble metal nanoparticles in a highly uniform size and shape distribution is of paramount importance.

Noble metal nanoparticles are typically synthesized by the reduction of precursor metal ions. Citrate-reduction of hydroauric acid $\left(\mathrm{HAuCl}_{4}\right)$ generally yields very homogeneous gold nanoparticles (AuNPs) with a monodisperse distribution of the size and shape. ${ }^{8}$ In this scheme, known as the Turkevich method, citrate functions as a surface stabilizer as well as a reductant. In marked contrast to AuNPs, citratereduction of silver ions produces extremely diverse silver nanoparticles (AgNPs) in sizes and shapes. ${ }^{9}$ This broad distribution of citrate-capped AgNPs impedes not only studies of size-dependent properties of AgNPs but also comparative studies between gold and silver nanoparticles with identical sizes and capping agents.

In this Note, we report a facile method of preparing uniform AgNPs using laser irradiation. Extensive studies have been done on the interactions of laser with AuNPs. Resonant excitation of the surface plasmon of AuNPs at 532 $\mathrm{nm}$ leads to fragmentation and melting of AuNPs. ${ }^{10-13}$ Relatively fewer reports are available on the laser irradiation of AgNPs. Notably, Pyatenko and coworkers found that at high laser powers $\left(532 \mathrm{~nm}, 200 \mathrm{~mJ} / \mathrm{cm}^{2}\right)$ AgNPs vaporize, producing Ag atoms, and then form core particles, which grow into $8 \mathrm{~nm}$-particles. ${ }^{14}$ They report that AgNPs remain unchanged at lower laser power. Here we recast the laser irradiation of AgNPs with a focus on preparing AgNPs with a monodisperse size distribution. Laser irradiation of a synthesized inhomogeneous mixture of AgNPs at $532 \mathrm{~nm}$, $80 \mathrm{~mJ} / \mathrm{cm}^{2}$ yields homogeneous spherical AgNPs with a diameter of $11.5 \mathrm{~nm}$, comparable to the size of AuNPs typically prepared by the Turkevich method; thus, these AgNPs can be used for comparative studies of noble metal nanoparticles with a similar size and surface characteristics, but differing in materials. As a demonstration, AgNPs, homogenized by the laser irradiation, are coated with silica $\left(\mathrm{Ag} @ \mathrm{SiO}_{2}\right.$ ) and compared with $\mathrm{Au} @ \mathrm{SiO}_{2}$.

\section{Experimental Method}

AgNPs were prepared using a method developed by Lee and Meisel, one of the most popular methods. ${ }^{15}$ An aqueous solution of $\mathrm{AgNO}_{3}(0.75 \mathrm{mM}, 100 \mathrm{~mL})$ was heated to boil and then trisodium citrate $(1 \% \mathrm{w} / \mathrm{v})$ was added with vigorous stirring. Continuing heating for $30 \mathrm{~min}$ yielded a turbid and greenish yellow solution of AgNPs, which was subsequently diluted by a factor of 10 . To remove the residual agents, the AgNP solution was centrifuged at 8000 $\mathrm{g}$ for $10 \mathrm{~min}$ and redispersed into water. The number of particles in $2.5 \mathrm{~mL}$ of sample is estimated at $6.5 \times 10^{9}$. All chemicals were purchased from Aldrich. Glassware was cleaned by aqua regia before use. AgNPs were characterized by UV-vis spectroscopy and transmission electron microscopy (TEM).

An Nd:YAG laser (GCR-11, Spectra-Physics) was used for irradiation. The second and third harmonic generation produces $532-\mathrm{nm}(300 \mathrm{~mW})$ and $355-\mathrm{nm}$ pulses $(150 \mathrm{~mW})$, respectively, at $10 \mathrm{~Hz}$. The laser beam was directed to a sample by two dichroic mirrors and used as unfocused (diameter $=7 \mathrm{~mm}$ ) to illuminate a large area of the sample in a quartz cell.

The changes in the size and shape of AgNPs induced by the laser irradiation were measured using a UV-vis spectrometer (Lambda 25, Perkin Elmer) and a transmission electron microscope (JEM-2100F, JEOL). The surface charges of AgNPs were measured using a zeta potential analyzer 
(Zetasizer Nano ZS, Malvern Instruments).

Silica coating of AgNPs and AuNPs was done employing a method described in a paper by Roca and Haes. ${ }^{16}$ Detailed procedures will be presented in a separate publication. ${ }^{17}$

\section{Results and Discussion}

Initially prepared AgNPs, following the Lee and Meisel method, exhibit a very broad UV-vis extinction band spanning from $350 \mathrm{~nm}$ to $700 \mathrm{~nm}$, reflecting diverse sizes and shapes (Figure 1(a)). The TEM image in Figure 1(b) confirms that the prepared AgNPs are indeed a mixture of various sizes and shapes of AgNPs. The size of AgNPs varies from $40 \mathrm{~nm}$ to $110 \mathrm{~nm}$. The shapes are diverse from spheres to rods and triangles, the distribution of which is presented as the pie graph in Figure 1 for 100 particles. It is known that spherical AgNPs with a diameter of $\sim 10 \mathrm{~nm}$ have an SPR band at $\sim 400 \mathrm{~nm}$ and, as the size increases, the

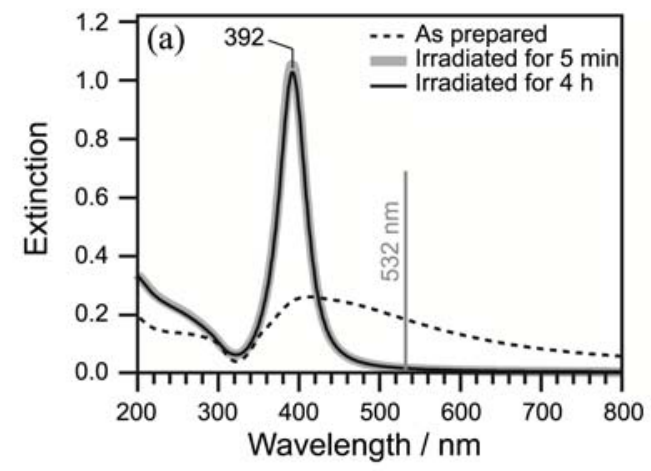

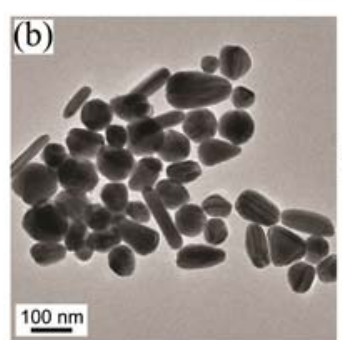

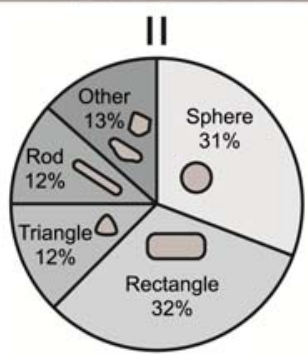

$(\mathrm{N}=100)$

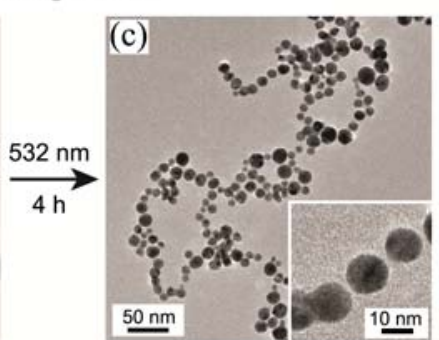

II

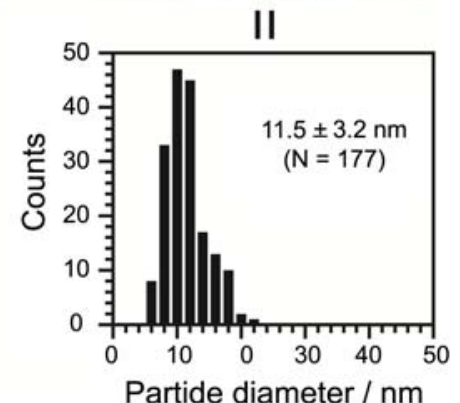

Figure 1. (a) UV-vis spectra of AgNPs, prepared by the Lee and Meisel method (dashed line) and after irradiation at $532 \mathrm{~nm}$ for 5 min (gray thick line) and for $4 \mathrm{~h}$ (solid line). TEM images of AgNPs (b) as prepared and (c) after irradiation at $532 \mathrm{~nm}$ for $4 \mathrm{~h}$. The inset in (c) represents a magnified image. The shape distribution of initially synthesized AgNPs is presented as the pie graph for 100 particles. Upon irradiation, all particles are transformed into spheres, of which size distribution is presented in the histogram.
SPR band redshifts. Metal nanorods have an additional extinction band in the longer wavelength region attributed to the longitudinal mode of surface plasmon. ${ }^{18}$

The broad UV-vis extinction band drastically changes upon irradiation at $532 \mathrm{~nm}$. Irradiation at $80 \mathrm{~mJ} / \mathrm{cm}^{2}$ for 5 min sharpens the band with $\lambda_{\max }$ at $392 \mathrm{~nm}$. Further extended irradiation to $4 \mathrm{~h}$ does not alter the spectrum significantly, as shown in Figure 1(a). This spectral change indicates that diverse shapes and sizes of AgNPs are transformed to homogenous spherical AgNPs by the laser irradiation at $532 \mathrm{~nm}$. TEM images are consistent with the spectral changes. Figure 1c shows that uniform spherical AgNPs with a size of $11.5 \pm 3.2 \mathrm{~nm}$ are produced by the irradiation.

Homogenization of AgNPs by the laser irradiation at moderate power can be explained by the resonant excitation of surface plasmon of large AgNPs, which leads to selective fragmentation of the nanoparticles. Excited surface plasmon of AgNPs eventually relaxes to phonons and solvents via electron-phonon coupling. ${ }^{19,20}$ During the relaxation process, fragmentation and melting of AgNPs occur. Laser irradiation at $532 \mathrm{~nm}$ selectively excites the surface plasmons of large Ag spheres, Ag nanorods, and Ag triangles, inducing the fragmentation and melting of those particles into smaller and spherical particles. Once the smaller and spherical AgNPs are produced, the surface plasmon absorption shifts to 392 $\mathrm{nm}$, where the 532-nm irradiation is not resonant any more. Thus, the AgNPs with a uniform size of 11-13 nm are maintained even if the irradiation continues.

When we switch the excitation wavelength from $532 \mathrm{~nm}$ to $355 \mathrm{~nm}$ after the homogeneous Ag nanospheres are produced, the UV-vis spectrum that has remained unchanged even with the continuing irradiation at $532 \mathrm{~nm}$ begins to change significantly. Figure 2 shows that the surface plasmon absorption band at $392 \mathrm{~nm}$ continually decreases with irradiation at $355 \mathrm{~nm}\left(40 \mathrm{~mJ} / \mathrm{cm}^{2}\right)$. After irradiation for $1 \mathrm{~h}$, the absorption band almost vanishes and the sample

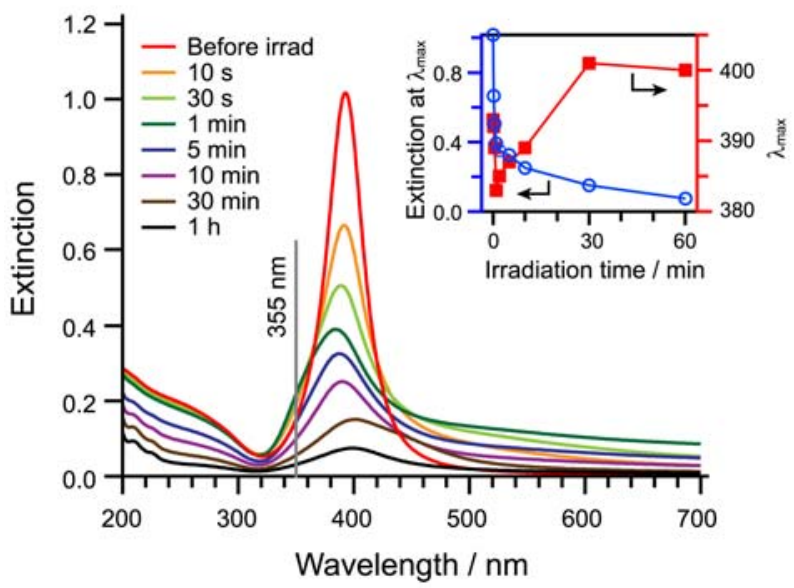

Figure 2. UV-vis spectra of AgNPs upon irradiation at $355 \mathrm{~nm}$ for the indicated period of time. The inset shows the plots of $\lambda_{\max }$ and extinction at $\lambda_{\max }$ as a function of the irradiation time. The used AgNPs were prepared by the Lee and Meisel method and then homogenized by the laser irradiation at $532 \mathrm{~nm}$ for $4 \mathrm{~h}$. 
becomes transparent. Resonant excitation of the surface plasmon of AgNPs at $355 \mathrm{~nm}$ completely fragments the particles.

A closer inspection into the spectral evolution with the irradiation time reveals that spherical AgNPs undergo fragmentation, followed by asymmetric growth to form nanowires, and then complete refragmentation. This process is consistent with the previously observed photoprocess of AuNPs. ${ }^{21}$ The inset in Figure 2 shows that, upon irradiation for $10 \mathrm{~s}$, the surface plasmon absorption band decreases and blueshifts, indicating that AgNPs are crumbled to smaller $\mathrm{Ag}$ nanoparticles and $\mathrm{Ag}$ atoms. Further irradiation up to 1 min leads to a continuing decrease and blueshift of the SPR band. In addition, a rise in the long wavelength region between 500 to $700 \mathrm{~nm}$ is observed. Consistently, the color of the sample appears black. As was observed for AuNPs, the rise in the long wavelength region is attributed to the formation of nanowires. ${ }^{21}$ TEM images show that nanowire networks are formed by the irradiation of $355 \mathrm{~nm}$ for $5 \mathrm{~min}$ (Supporting Information). We believe that the uneven distribution of surface capping agents (i.e., citrate anions) on the fragmented AgNPs causes dipole-induced asymmetric growth. The Ag nanowires are then fragmented by the extended irradiation (30 min and $60 \mathrm{~min}$; see also Supporting Information). These results confirm that off- and onresonance between the surface plasmon of AgNPs and the excitation wavelength play a key role in the homogenization and shape changes of AgNPs.

We found that the AgNPs prepared by the laser irradiation at $532 \mathrm{~nm}$ as described above, despite their improved uniformity, were not appropriate for a long-term use or storage. Figure 3 shows that surface plasmon absorption at $392 \mathrm{~nm}$ decreases on a time scale of days, indicating that the AgNPs are unstable and slowly precipitate by coagulation. This is probably due to the increased surface area of the AgNPs when fragmented by the laser irradiation. The total

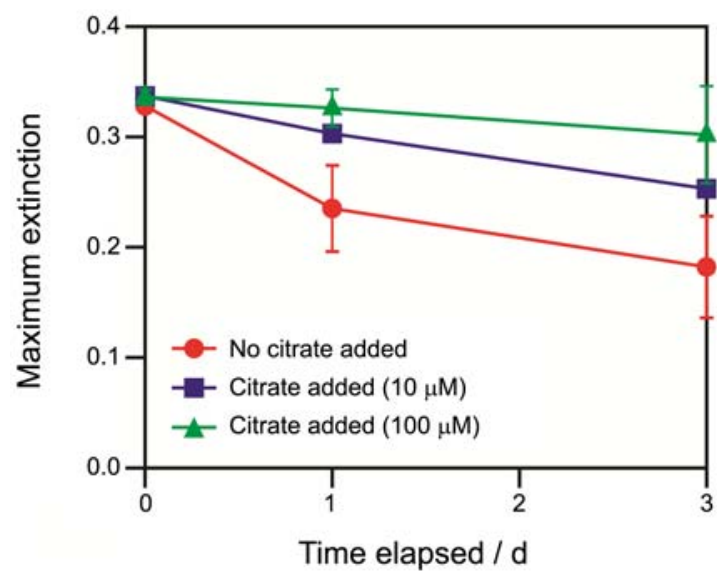

Figure 3. Stability of AgNPs homogenized by the laser irradiation at $532 \mathrm{~nm}$ for $4 \mathrm{~h}$ (red circles). The extinction at $392 \mathrm{~nm}$ was measured as time elapses after the termination of irradiation. Blue squares and green triangles represent the change of the extinction of the homogenized AgNP samples where citrate was added to 10 and $100 \mu \mathrm{M}$, respectively. (a) $\mathrm{Ag} @ \mathrm{SiO}_{2}$

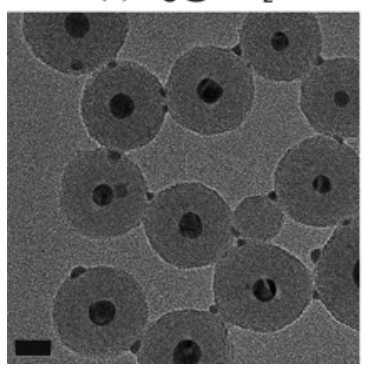

(b) $\mathrm{Au} @ \mathrm{SiO}_{2}$

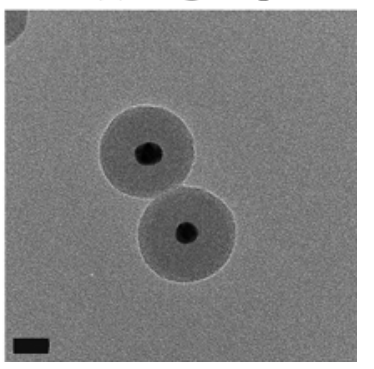

Figure 4. (a) Silica-coated AgNPs $\left(\mathrm{Ag} @ \mathrm{SiO}_{2}\right)$ and (b) silicacoated AuNPs $\left(\mathrm{Au} @ \mathrm{SiO}_{2}\right)$. AgNPs were prepared by the laser irradiation at $532 \mathrm{~nm}$, as described in the text. AuNPs were synthesized using the Turkevich method. Scale bars indicate 20 nm.

surface area increases by a factor of 7 when 80-nm Ag nanospheres are converted to $12-\mathrm{nm}$ ones. Therefore, the amount of stabilizing agents (in this case, citrate) used to stabilize the larger AgNPs is just not enough to cover the increased surface areas of smaller AgNPs. The reduced surface charges of smaller AgNPs due to deficient citrate on the surface are confirmed by the low magnitude of zeta potential, $-31 \pm 1$ $\mathrm{mV}$. We added an extra amount of citrate anions to compensate the shortage of the surface stabilizing agents after the completion of irradiation for $4 \mathrm{~h}$. Figure 3 shows that addition of citrate to a final concentration of 10 and $100 \mu \mathrm{M}$ increasingly improves the stability of AgNPs. The zeta potential also increases to $-51 \pm 2 \mathrm{mV}$ when citrate is added to $100 \mu \mathrm{M}$. These results suggest that irradiation at $532 \mathrm{~nm}$, followed by the addition of citrate, is an effective method of preparing stable and monodisperse AgNPs.

The AgNPs prepared by this method are comparable to the AuNPs prepared by the Turkevich method in sizes $(\sim 13 \mathrm{~nm})$ and surface stabilizing agents (citrate). Similar structural and surface characteristics of the two types of noble metal nanoparticles allow for investigations of various materialdependent properties without concern about other factors. As a demonstration, we fabricated silica-coated $\mathrm{Au}$ and $\mathrm{Ag}$ nanoparticles (Figure 4). Citrate-capped monodisperse AgNPs $(12.6 \pm 2.1 \mathrm{~nm})$ were prepared by the laser irradiation as discussed above and then encapsulated by silica shells with a thickness of $20 \mathrm{~nm}$ by employing exactly the same method applied to the AuNPs $(13.3 \pm 0.7 \mathrm{~nm})$ synthesized by the Turkevich method. ${ }^{16}$ Comparison between the two coreshell nanostructures will provide insights into the materialdependent surface plasmon properties such as SERS and surface plasmon couplings.

In conclusion, we found that the pulsed-laser irradiation at $532 \mathrm{~nm}$ at a moderate power can be effectively used to prepare citrate-capped AgNPs with a monodisperse size distribution. Conventional citrate reduction of $\mathrm{Ag}^{+}$usually yields diverse AgNPs in sizes and shapes. Irradiation of the prepared AgNPs at $532 \mathrm{~nm}\left(80 \mathrm{~mJ} / \mathrm{cm}^{2}\right)$ converts the various shapes and sizes of AgNPs to homogeneous and uniform $\mathrm{Ag}$ nanospheres with a diameter of $\sim 12 \mathrm{~nm}$. These structural changes sharpen the broad surface plasmon absorption band 
to the narrow band centered at $392 \mathrm{~nm}$. Further irradiation at $355 \mathrm{~nm}$ leads to complete fragmentation of the Ag nanospheres. Resonant excitation of surface plasmon explains the wavelength-dependent changes and homogenization of the AgNPs. Addition of slight amounts of citrate stabilizes the AgNPs for a long-term use or storage. Laser-induced homogenization of AgNPs presented here provides a simple route to the preparation of monodisperse AgNPs capped with citrate.

\section{References}

1. Odom, T. W.; Pileni, M.-P. Acc. Chem. Res. 2008, 41, 1565.

2. Kelly, K. L.; Coronado, E.; Zhao, L. L.; Schatz, G. C. J. Phys. Chem. B 2003, 107, 668.

3. Jain, P. K.; Lee, K. S.; El-Sayed, M. A. J. Phys. Chem. B 2006, $110,7238$.

4. Moskovits, M. J. Raman Spectrosc. 2005, 36, 485.

5. Michaels, A. M.; Nirmal, M.; Brus, L. E. J. Am. Chem. Soc. 1999, $121,9932$.

6. Cao, Y. C.; Jin, R.; Mirkin, C. A. Science 2002, 297, 1536.

7. Jain, P. K.; Huang, X.; El-Sayed, I. H.; El-Sayed, M. A. Acc. Chem. Res. 2008, 41, 1578.
8. Turkevich, J.; Stevenson, P. C.; Hillier, J. Discuss. Faraday Soc. $1951,11,55$.

9. Evanoff, D. D.; Chumanov, G. ChemPhysChem 2005, 6, 1221.

10. Link, S.; Burda, C.; Mohamed, M. B.; Nikoobakht, B.; El-Sayed, M. A. J. Phys. Chem. A 1999, 103, 1165.

11. Link, S.; Burda, C.; Nikoobakht, B.; El-Sayed, M. A. J. Phys. Chem. B 2000, 104, 6152.

12. Mafuné, F.; Kohno, J.-y.; Takeda, Y.; Kondow, T. J. Phys. Chem. $B$ 2001, 105, 9050.

13. Mafuné, F.; Kohno, J.-y.; Takeda, Y.; Kondow, T. J. Phys. Chem. B 2003, 107, 12589.

14. Pyatenko, A.; Yamaguchi, M.; Suzuki, M. J. Phys. Chem. B. 2005, 109, 21608.

15. Lee, P. C.; Meisel, D. J. Phys. Chem. 1982, 86, 3391.

16. Roca, M.; Haes, A. J. J. Am. Chem. Soc. 2008, 130, 14273.

17. Lee, S.; Yoon, S. 2011, in preparation.

18. Murphy, C. J.; Sau, T. K.; Gole, A. M.; Orendorff, C. J.; Gao, J.; Gou, L.; Hunyadi, S. E.; Li, T. J. Phys. Chem. B 2005, 109, 13857.

19. Ahmadi, T. S.; Logunov, S. L.; El-Sayed, M. A. J. Phys. Chem. 1996, 100, 8053.

20. Hodak, J. K.; Martini, I.; Hartland, G. V. J. Phys. Chem. B 1998, 102, 6958.

21. Park, J. S.; Yoon, J. H.; Kim, H. J.; Huh, Y.-D.; Yoon, S. Bull. Korean Chem. Soc. 2010, 31, 819. 